\title{
Breaking Barriers: Prospective Study of a Cohort of Advanced Chronic Obstructive Pulmonary Disease Patients To Describe Their Survival and End-of-Life Palliative Care Requirements
}

\author{
Daniel Gainza-Miranda, MD, ${ }^{1}$ Eva Maria Sanz-Peces, RN, ${ }^{1}$ Alberto Alonso-Babarro, PhD, ${ }^{2}$ \\ María Varela-Cerdeira, MD, ${ }^{2}$ Concepción Prados-Sánchez, PhD, ${ }^{3}$ Guadalupe Vega-Aleman, MD, ${ }^{4}$ \\ Ricardo Rodriguez-Barrientos, $\mathrm{PhD}^{5}$ and Elena Polentinos-Castro, $\mathrm{PhD}^{5}$
}

\begin{abstract}
Background and Aim: Consensus has been reached on the need to integrate palliative care in the follow-up examinations of chronic obstructive pulmonary disease (COPD) patients. We analyzed the survival from the initiation of follow-up by a palliative home care team (PHCT) and described the needs and end-of-life process. Setting and Design: This study was a prospective observational cohort study of advanced COPD patients referred to a PHCT. Sociodemographic variables, survival from the start date of follow-up using the KaplanMeier model, health resource consumption, perceived quality of life, main symptomatology, opioid use, and advanced care planning (ACP) were analyzed.

Results: Sixty patients were included. The median survival was 8.3 months. Forty-two patients died at the end of the study (85\% at home or in palliative care units). The most frequent cause of death was respiratory failure in 39 patients (93\%), with 29 of these patients requiring sedation (69\%). Dyspnea at rest, with an average of 5 (standard deviation [SD] 2) points, was the main symptom. Fifty-five patients (91\%) required opioids for symptom control. The median score in the St. George's Respiratory Questionnaire was 72 (SD 13). The mean number of visits by the home team was 7 (SD 6.5). The mean number of admissions during the monitoring period was 1.5 (SD 0.15).

Conclusions: The characteristics of the cohort appear suitable for a PHCT. The follow-up care provided by our multidisciplinary unit decreased the number of hospitalizations, favored the development of ACP, and enabled death at home or in palliative care units.
\end{abstract}

Keywords: ACP; end of life; palliative care; place of death; pulmonary disease chronic obstructive; quality of life

\section{Introduction}

$\mathbf{C}$ HRONIC OBSTRUCTIVE PULMONARY DISEASE (COPD) is currently the fourth leading cause of death in Western countries. ${ }^{1}$

The advanced stage of the disease is characterized by a high frequency of symptoms, loss of functionality, and a great number of exacerbations, leading to a significant deterioration in the patient's quality of life. This deterioration is similar or even higher than the final stage of advanced cancer patients. ${ }^{2-4}$ However, access to specific palliative care resources for COPD patients remains low., ${ }^{5,6}$ The literature indicates that multiple barriers are observed in the access of these patients to palliative care. ${ }^{7}$ A proper description of the

\footnotetext{
${ }^{1}$ Palliative Homecare Team Northern Area of Madrid, SERMAS, San Sebastian de los Reyes, Spain.

${ }^{2}$ Palliative Care Department and ${ }^{3}$ Pneumology Department, La Paz Hospital, Madrid, Spain.

${ }_{5}^{4}$ Preventive Medicine, Public Health, SERMAS, San Sebastian de los Reyes, Spain.

${ }^{5}$ Research Support Unit, Primary Care Management, Madrid Health Service; Research Network in Health Services and Chronic Diseases (REDISSEC), Madrid, Spain.

Accepted October 5, 2018.

(C) Daniel Gainza-Miranda et al., 2018; Published by Mary Ann Liebert, Inc. This Open Access article is distributed under the terms of the Creative Commons License (http://creativecommons.org/licenses/by/4.0), which permits unrestricted use, distribution, and reproduction in
} any medium, provided the original work is properly credited. 
final phase of this disease may allow for a better assessment of patients' needs and their care and treatment. ${ }^{8}$

The difficulty in establishing the prognosis and recognition of the final stage of life may represent one of the main causes of nonreferral to palliative care services. ${ }^{7,9}$ Therefore, the main consensus guidelines for the management of COPD patients include the collection of prognostic criteria to allow for the identification of end-of-life patients. ${ }^{10-12}$ Other authors recommend starting an early palliative care approach for the disease regardless of its prognosis. ${ }^{13}$

Patients with advanced COPD are generally in a home environment and require hospital care during flare-ups. Pneumology, palliative care, and primary care departments should work in a coordinated manner to ensure the end-of-life continuity of care. However, there barely exist works on the most effective health structure for attending to end of life for these patients, nor on the natural background of patients with advanced COPD. ${ }^{14,15}$ These kinds of studies can provide us with information about symptomatic burden, quality of life, and real needs of health resources for these patients. This information would let us develop more effective health structures and improve the care that these patients receive, offering them and their families more realistic expectations as the disease progresses and they approach the moment of death.

A multidisciplinary unit for the care of patients with advanced respiratory problems that integrated the pneumology department and palliative care unit was created in our hospital in 2013. Residential and hospital services are included in the palliative care unit, which are responsible for contact with the primary care doctors. The HOLD study ${ }^{16}$ sought to describe the trajectory at the end of life in actual clinical practice in patients seen by a palliative home care team (PHCT) integrated into a multidisciplinary unit, and the results allow for measures to be taken to improve the care model.

We analyzed the survival from the start of monitoring by a PHCT that had been in place for four years and described the end-of-life needs and processes of these patients. Other specific goals of this work were as follows: to describe the main symptoms and quality of life, to estimate the use of opioids in standard clinical practice for treating dyspnea, and to evaluate the use of health resources in advanced stages of COPD patients monitored at home.

\section{Materials and Methods}

\section{Design}

This study was a prospective observational cohort study of advanced COPD patients referred by the pneumology department, palliative care support team, or primary care team for monitoring by the PHCT.

The inclusion criteria were as follows: older than 18 years, COPD Global Initiative for Chronic Obstructive Lung Disease (GOLD) grade IV, ${ }^{11}$ and functional deterioration with a palliative performance scale (PPS) $<60$. The exclusion criteria were as follows: cognitive impairment or severe mental illness, a diagnosis of lung cancer or cystic fibrosis, inability to receive home care because of the absence of a primary caregiver and inability to understand Spanish.

Patients diagnosed with lung cancer during the monitoring period or those who moved out of the study area were also excluded. All patients provided written consent for their participation in the study.

Once a patient was included in our program, monthly monitoring visits up to a two-year maximum were carried out by the PHCT in addition to the usual clinical care. In case of urgent hospital admission, the attendance of the patients in the program was carried out by a support team or the pneumology service, both included in the multidisciplinary unit, to guarantee continuity of care. During the follow-up, the palliative care unit could request a hospice admission if deemed necessary. Other PHCT key components are shown in Table 1. The monitoring protocol and the scope of the study were extensively described in a previous work by our group (HOLD study). ${ }^{16}$

The ethics committee approved the study with the project code Pi-2011.

\section{Variables}

The survival time of the patient cohort from the inclusion in the study to the date of death was calculated as the main variable. The date and place of death, reason for death, need for palliative sedation (defined as the use of specific sedatives to relieve intolerable suffering from refractory symptoms by reducing a patient's level of consciousness), ${ }^{17}$ clinical reason for sedation, and the implementation of advanced care planning (ACP) were recorded for the end-of-life description.

The following secondary variables were studied.

Sociodemographic variables. Data on age, gender, and education level were recorded at the start of monitoring.

Variables related to clinical characteristics. The following data were recorded: forced expiratory volume in one second (FEV1) in last spirometry, use of chronic domiciliary oxygen therapy and noninvasive mechanical ventilation

Table 1. Palliative Home Care Team Key COMPONENTS IN THE MUlTIDISCIPLINARY UNIT

1. Attendance at monthly meetings of the multidisciplinary unit to agree on treatments and care plans for new patients and update treatment goals for patients in the program.

2. Monthly scheduled domiciliary visits by PHCT and telephone support and nonscheduled domiciliary visits as needed.

3. Primary care support with joint domiciliary visits and telephone consultations.

4. Disease treatment optimization, including education and management of inhaler therapy, domiciliary oxygen therapy, and written exacerbation plans.

5. Holistic and systematic assessment of symptoms with special attention to dyspnea with comprehensive management of refractory breathlessness, including nonpharmacological strategies (such as breathing techniques, recovery breathing positions, and the use of a handheld fan) and written instructions for the use of opioids prescribed.

6. Early access to hospice services to avoid hospital or emergency department admissions.

7. Routine discussion regarding goals of care and advanced care planning.

PHCT, palliative home care team. 
(NIMV), number of admittances in the year before the start of monitoring, BODE Index, ${ }^{18}$ Charlson Comorbidity Index, ${ }^{19}$ PPS, ${ }^{20}$ Barthel Index (BI), ${ }^{21}$ body mass index, and level of physical activity using the short version of the International Physical Activity Questionnaire. ${ }^{22}$

Quality of life. St. George's Respiratory Questionnaire (SGRQ) was self-administered and supervised on the first visit to the patient's home and quarterly during the follow-up. ${ }^{23}$

Symptom load and care. The Edmonton Symptom Assessment System (ESAS) ${ }^{24}$ and the dyspnea grade according to the Modified Scale of the Medical Research Council (mMRC) were recorded at every home visit. ${ }^{25}$ The percentage of patients with an opioid prescription during monitoring to control dyspnea and the average doses were also recorded.

The number of visits by the PHCT and phone calls made to the team by patients or their families during the monitoring period was determined via clinical records. We also collected the numbers of visits to the emergency department (ED) and hospital admissions during the year before the start of monitoring and during the monitoring.

\section{Statistical analysis}

Most of the objectives were described via descriptive statistics that summarized the categorical variables using absolute and relative frequencies. Continuous variables with a normal distribution were analyzed using the mean and standard deviation (SD), and continuous variables with an asymmetrical distribution were analyzed using the medians and interquartile ranges (IQR). The survival curves were generated using the Kaplan-Meier model. The survival assessment was reported with a confidence interval of $95 \%$ (95\% CI). Dependent $t$-test was used to evaluate the difference in admittances and visits to the ED before and after the start of the follow-up by PHCT.

The statistical program used was Stata (StataCorp.2013. Stata Statistical Software: Release 13; StataCorp LP, College Station, TX).

\section{Results}

A total of 66 advanced COPD patients were referred to the PHCT between January 1, 2014, and February 1, 2017. Six patients were excluded because they did not meet the inclusion criteria (five due to PPS $>60$ and one due to of GOLD grade III). Five of the 60 included patients $(8 \%)$ were discharged during monitoring because of functional improvement, and one patient refused to continue in the study.

Most of the patients in our study were males (48). The mean age was 73 years (SD 12). The FEV1 was 26.8 (SD 4.3). Table 2 shows the remaining demographic and clinical characteristics at the start of monitoring.

The median survival of patients from the start of monitoring by the unit was 8.3 months (95\% CI 2.7-20.5) (Fig. 1). A total of $42(70 \%)$ patients died. Thirty-six of these patients $(85 \%)$ died at home or in palliative care units (Fig. 2). The cause of death was respiratory failure in 39 patients $(93 \%)$ and complications related to comorbidity in the remaining 3 patients (7\%). Twenty-nine patients $(69 \%)$ required pal-
Table 2. Demographic and Clinical Variables at The Start of Monitoring

\begin{tabular}{lc}
\hline Variable (n=60) & Total \\
\hline Age: mean (SD) & $73.8(12.2)$ \\
Gender: male (\%) & 80 \\
Level of studies (\%) & 6.6 \\
Without studies & 20 \\
Vocational training & 48.3 \\
Primary & 11.6 \\
Secondary & 13.3 \\
University & $26.8(4.3)$ \\
FEV1 (SD) & $6(3.8)$ \\
BODE score (\%) & $7(27)$ \\
& $8(11.5)$ \\
& $9(23.1)$ \\
& $10(34.6)$ \\
Physical activity (\%) & 5 \\
Medium & 95 \\
Low/sedentary & 31.6 \\
NIMV (\%) & $2.5(1.57) / 1.5$ \\
No. of admittances in the year before & $(0.15), p<0.01$ \\
the start of monitoring/after & \\
monitoring: mean (SD) & $3.5(2.01) / 0.8$ \\
Number of visits to the emergency & $(1.04), p<0.01$ \\
department in the year before the & \\
start of monitoring/after & $22(4.4)$ \\
monitoring: mean (SD) & $<21(42)$ \\
PPS: mean (SD) & $51.66(9.7)$ \\
Barthel Index: mean (SD) & $69.41(24.8)$ \\
Charlson Index: mean (SD) & $2.5(1.65)$ \\
Home oxygen therapy prescription $(\%)$ & 100 \\
BMI (SD) & \\
BMI <21 (\%) & \\
\hline
\end{tabular}

BMI, body mass index; FEV1, forced expiratory volume in one second; NIMV, noninvasive mechanical ventilation; PPS, palliative performance scale; SD, standard deviation.

liative sedation, including in the hospital and home area. Dyspnea was the main cause for palliative sedation in 24 patients $(83 \%)$. Other reasons were agitation in three patients $(10 \%)$ and existential distress in two patients $(7 \%)$.

At the beginning of the follow-up, none of the 42 deceased patients had performed ACP. During the follow-up, 23

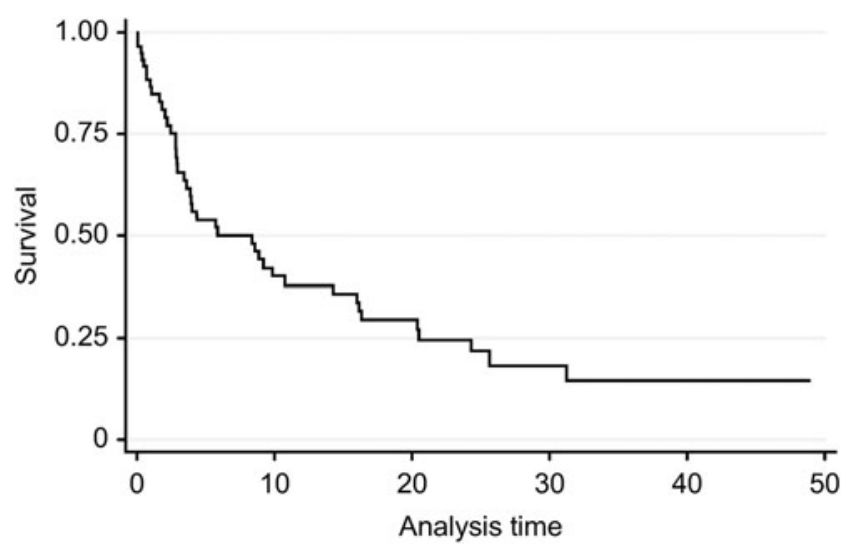

FIG. 1. Kaplan-Meier survival curve. 


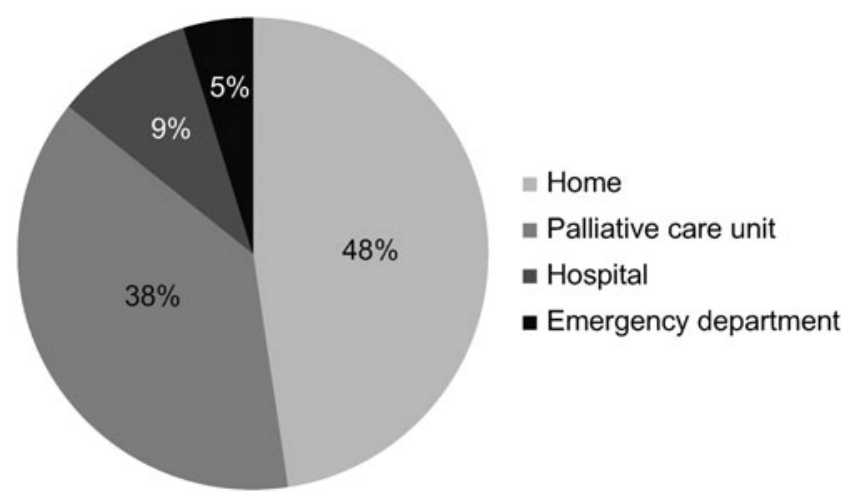

FIG. 2. Place of death.

patients $(55 \%)$ had performed ACP. Nine patients $(21 \%)$ did not perform ACP due to the short time of follow-up (less than three visits), while eight patients (19\%) did not wish to discuss ACP and two patients $(5 \%)$ did not perform ACP due to conspiracy of silence.

Fifty-four patients (90\%) had grade IV dyspnea according to the mMRC, and the remaining six $(10 \%)$ patients had dyspnea grade III. Dyspnea at rest was the symptom with the highest score in the ESAS. Main results of ESAS and SGRQ during the follow-up are summarized in Figure 3.

Fifty-five $(91 \%)$ patients required opioids to control their dyspnea, and morphine was the opioid used in 54 cases (90\%). The mean equivalent daily dose of morphine (EDDM) was $14.88 \mathrm{mg}$ (SD 14) of basal opioids, and the mean rescue EDDM was $6.21 \mathrm{mg}$ (SD 12.5). At the dying phase, the mean EDDM was $44.8 \mathrm{mg}$ (SD 16.5).

The median number of visits by the PHCT was 4.5 (IQR 2-20). Forty-nine patients (82\%) made calls to the PHCT, and the median number of calls from these patients was 4 (IQR 2-6).

The mean number of admittances and emergency visits in the year before the monitoring and after monitoring is shown in Table 2.

\section{Discussion}

The HOLD study is one of the first cohort studies of patients with advanced COPD who were followed by a PHCT. Our results show that our chosen inclusion criteria are feasible for joint monitoring by palliative care teams according to the high symptom load and a limited life expectancy showed by our cohort.

The profiles of the patients in the unit are consistent with the profile of patients who were considered in end-of-life stage by the European Respiratory Society ${ }^{9}$ and the descriptions of end-of-life profiles in different publications. ${ }^{12}$ The survival rate of the cohort was 8.3 months. These patients were subsidiary for follow-up by specific palliative care teams according to the literature. ${ }^{7,9,26,27}$ Different published reviews indicated that early palliative care should be started in parallel to active treatment and monitoring by specific teams must be requested when uncontrolled symptoms are observed and for ACP, which must be preferably performed in the final stages of the disease. ${ }^{28,29}$

In a study similar to ours conducted in Canada, Horton et al. ${ }^{30}$ published results on the intervention of a palliative care team in a cohort of patients with moderate or severe COPD. The profile of the 30 recruited patients revealed an important degree of obstruction with a dyspnea grade that was somewhat lower than that of our cohort (resting dyspnea in $60 \%$ of patients), and home oxygen therapy was observed in half of their patients. The study did not offer a survival median, although half of the patients died after one year of monitoring and exhibited a symptom load and deterioration of quality of life similar to that in our study.

In another study conducted in Australia, Smallwood et al. ${ }^{15}$ published results of a cohort of 171 patients with advanced COPD followed up by the integrated respiratory and palliative care team. Patients also revealed an important degree of obstruction and minimal exertion dyspnea in $75 \%$ of patients. Sixty-two patients of the cohort died with a survival median of 12.1 months (7.8-27.1). No symptom control or quality-of-life measures were reported.

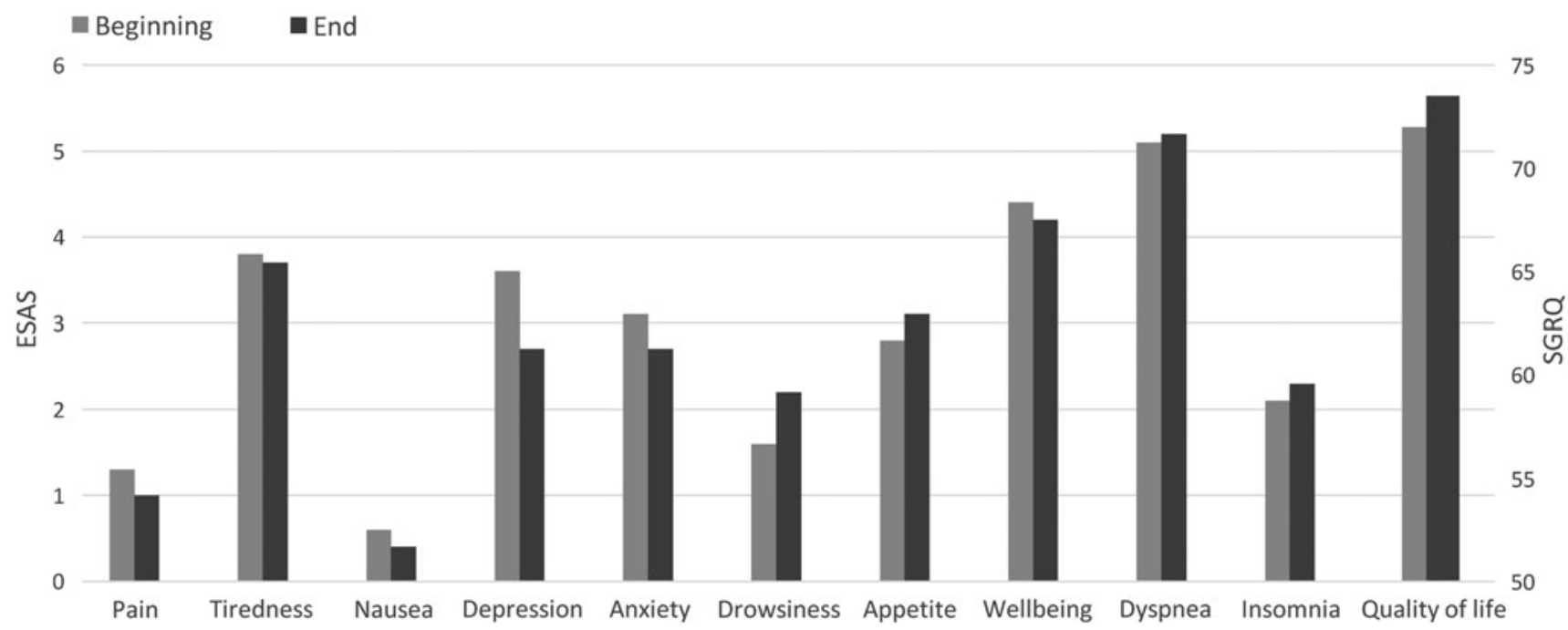

FIG. 3. Evolution of symptoms and quality of life. ESAS, Edmonton Symptom Assessment System; SGRQ, St. George's Respiratory Questionnaire. 
The main cause of death in our patients was respiratory insufficiency, which was followed to a much lesser degree by secondary complications to the comorbidity. However, in many studies, patients with COPD generally die because of comorbidity, especially morbidity caused by cardiovascular causes and cancer. ${ }^{31,33}$ This result may be explained because the comorbidity of our cohort was not very high, which was likely because most of the patients were included from the pneumology outpatient clinics, where the patient profile may be different from that in other areas. However, in studies focused on populations with advanced COPD, the main cause of death is respiratory failure, which was also the case in our study. ${ }^{34,35}$ Dyspnea was the main symptom listed at the first visit, and the diagnosis was primarily minimal effort or rest dyspnea. Tiredness, loss of well-being, and depression were other prevalent symptoms in our cohort. These findings are consistent with most studies published on advanced COPD patients. ${ }^{36,37}$ Most patients required the use of opioids to control dyspnea during monitoring. The doses used were adjusted to the recommendations in terms of safety for the control of dyspnea in patients with advanced COPD $^{38}$ and were similar to that of previous studies. ${ }^{39,40}$ In contrast, several studies show the underuse of opioids among pneumologists. $^{41,42}$

High scores in the SGRQ were obtained at the start and similar results were found after three months of follow-up despite the progression of disease. Our results in terms of loss of quality of life were far superior to that of other cohort studies of patients with COPD. ${ }^{43}$ This difference was likely related to performance in patients at earlier stages of the disease.

The main places of death were home and palliative care unit (PCU), which covered $85 \%$ of deaths. These results are consistent with the results of Smallwood et al., in which only $24.6 \%$ of patients died in an acute hospital bed. Boland et al. ${ }^{44}$ also found that $63 \%$ of patients died at home or in the PCU, which was somewhat lower than that of our study.

A recent study that analyzed the place of death in 14 countries $^{45}$ found that the percentage of death at home ranged from $54 \%$ in Mexico to $10.4 \%$ in Canada. Spain exhibited an intermediate percentage (36\%). The differences are explained by the presence of a primary caregiver, the monitoring by palliative care teams, the development of ACP, age, and cultural factors that favor death at home. These data together with a different organization of patient monitoring may explain our higher percentage of deaths in the home and PCU.

None of our patients had performed ACP before referral. However, ACP was undertaken in half of the patients during the follow-up. These data are inconsistent with several studies, ${ }^{15,46}$ where communication with end-of-life patients with COPD was reported as poor. In contrast, Sinclair et al. ${ }^{47}$ systematically performed end-of-life conversations and obtained percentages similar to our study. The undertaking of ACP is facilitated by participation in the monitoring of palliative care teams as previously described. ${ }^{48}$ Earlier referral to the palliative care team could improve percentage of ACP in the future as nine patients $(21 \%)$ with no ACP had a less than three-month follow-up. We also found that eight patients (19\%) did not wish to discuss ACP. Similar percentages were also found in the literature. ${ }^{49}$

A total of $69 \%$ of patients required palliative sedation. To ensure appropriate use of palliative sedation, the PHCT fol- lowed a previously created checklist. ${ }^{17}$ Our group and others have described a much lower percentage of patients requiring palliative sedation at home. ${ }^{17}$ However, data are not available for sedation at the end of life in patients with COPD. A systematic review of studies on sedation at home that included almost exclusively oncological patients obtained percentages between $1 \%$ and $72 \% .{ }^{50} \mathrm{~A}$ high frequency of symptoms of poor control justifies the higher percentages of sedation. ${ }^{51}$ Caraceni et al. ${ }^{52}$ performed a retrospective study in a tertiary Italian hospital and obtained a rate of $68 \%$ sedation, which was similar to our own results. The presence of dyspnea as the most prevalent symptom and the main reason of sedation in our study may help explain our high percentage of sedation.

The literature describes that the number of hospitalizations and ED visits increases with the approach of end of life of COPD patients. ${ }^{53}$ This increase was not observed in our study, and the number actually decreased from the previous year. This result may be related with the involvement of specific palliative care teams in patient monitoring, as it was reported by at least two systematic reviews. ${ }^{54,55}$

Our study has several limitations. First, most of the patients in the study were included from pneumology consultations, which could affect certain results. Nevertheless, the inclusion of patients from other areas would not change the main conclusions of our study. Second, this was also a small, long-term, single-group, cohort study and so there was no separate control group, which may limit the generalization of the results. There are no previous studies that describe the end of life in the home setting with which we can compare our results. A "before and after" comparison was undertaken to investigate any associations between PHCT care and unscheduled health care use. Future cluster multicenter randomized studies should be done to rigorously test the effectiveness of the PCHT follow-up.

Therefore, we conclude that the patient profile of our PHCT had a survival of eight months, exhibited a high symptom load, and presented a low quality of life during the follow-up and low levels of physical and functional activity, consistent with the profile of patients who require the attention of a specific palliative care team. Monitoring by our PHCT appears to favor the development of ACP and a smaller number of hospitalizations to enable death at home or in palliative care units.

\section{Acknowledgments}

The authors thank the ISCIII, FEDER funds, and FIIBAP for the funding received and the Madrid Primary Care Research Unit for its technical support. They also thank Juan Jose Ruiz Martinez for his informatic support.

Funding: The HOLD study is funded by a grant from the Fund for Health Research (FIS) under project number PI14/ 01271 through the Carlos III Health Institute (ISCIII) and the FEDER funds. The study also received an intensification grant provided by the Foundation for Biomedical Research and Innovation of Primary Care (FIIBAP).

\section{Author Disclosure Statement}

No competing financial interests exist. 


\section{References}

1. WHO: World Health Report. Geneva: World Health Organisation, 2003. http://who.int/whr/2003/en/ (last accessed October 5, 2018).

2. Claessens MT, Lynn J, Zhong Z, et al.: Dying with lung cancer or chronic obstructive pulmonary disease: Insights from SUPPORT. Study to understand prognoses and preferences for outcomes and risks of treatments. J Am Geriatr Soc 2000;48(5 Suppl):S146-S153.

3. Bausewein C, Booth S, Gysels M, et al.: Understanding breathlessness: cross-sectional comparison of symptom burden and palliative care needs in chronic obstructive pulmonary disease and cancer. J Palliat Med 2010;13: 1109-1118.

4. Romem A, Tom SE, Beauchene M, et al.: Pain management at the end of life: A comparative study of cancer, dementia and chronic obstructive pulmonary disease patients. Palliat Med 2015;29:464-469.

5. Wachterman MW, Pilver C, Smith D, et al.: Quality of endof-life care provided to patients with different serious illnesses. JAMA Intern Med 2016;176:1095-1102.

6. Burt J, Shipman C, Richardson A, et al.: The experiences of older adults in the community dying from cancer and noncancer causes: A national survey of bereaved relatives. Age Ageing 2010;39:86-91.

7. Harrington SE, Rogers E, Davis M: Palliative care and chronic obstructive pulmonary disease. Curr Opin Pulm Med 2016;23:1.

8. Callahan D: Death and the research imperative. N Engl J Med 2000;342:654-656.

9. Curtis JR: Pallliative and end-of-life care for patients with severe COPD. Eur Respir J 2008;32:796-803.

10. Lanken PN, Terry PB, DeLisser HM, et al.: On behalf of the ATS end-of-life care task force. An official American Thoracic Society clinical policy statement: Palliative care for patients with respiratory diseases and critical illnesses. American Thoracic Society Documents. Am J Respir Crit Care Med 2008;177:912-927.

11. Global Strategy for the Diagnosis, Management and Prevention of COPD, Global Initiative for Chronic Obstructive Lung Disease (GOLD) 2017. http://goldcopd.org (last accessed October 5, 2015).

12. Miravitlles M, Soler-Cataluña JJ, Calle M, et al.: Spanish Guideline for COPD (GesEPOC). Update 2014. Arch Bronconeumol 2014;50(Supl 1):1-16.

13. Bourke SJ, Peel ET: Palliative care of chronic progressive lung disease. Clin Med 2014;14:79-82.

14. Boland J, Martin J, Wells AU, Ross JR: Palliative care for people with non malignant lung disease: Summary of current evidence and future direction. Palliat Med 2013;27:811-816.

15. Smallwood N, Thompson M, Warrender-Sparkes M, et al.: Integrated respiratory and palliative care may improve outcomes in advanced lung disease. ERJ Open Res 2018;4:102-2017.

16. Gainza Miranda D, Sanz Peces EM, Alonso Babarro A, et al.: HOLD study (Home care Obstructive Lung Disease): Natural history of patients with advanced COPD. BMC Palliat Care 2016;15:35.

17. Alonso-Babarro A, Varela-Cerdeira M, Torres-Vigil I, et al.: At-home palliative sedation for end-of-life cancer patients. Palliat Med 2010;24:486-492.

18. Celli BR, Cote CG, Marin JM, et al.: The bodymass index, airflow obstruction, dyspnea, and exercise capacity index in chronic obstructive pulmonary disease. N Engl J Med 2004; 350:1005-1012.
19. Charlson M, Pompei P, Ales KL, McKenzie CR: A new method of classyfing prognostic comorbidity in longitudinal studies: Development and validation. J Chronic Dis 1987;40:373-383.

20. Anderson F, Downing GM, Hill J, Casorso L, Lerch N: Palliative performance scale (PPS): A new tool. J Palliat Care 1996;12:5-11.

21. Mahoney FI, Barthel DW: Functional evaluation: The Barthel Index. Md State Med J 1965;14:61-65.

22. Craig CL, Marshall AL, Sjöström M, et al.: International physical activity questionnaire: 12-country reliability and validity. Med Sci Sports Exerc 2003;35:1381-1395.

23. Jones PW, Quirk FH, Baveystock CM: The St George's respiratory questionnaire. Respir Med 1991;85 Suppl B:2531, discussion 33-37.

24. Bruera E, Kuehn N, Miller MJ, et al.: The Edmonton Symptom Assessment System (ESAS): A simple method for the assessment of palliative care patients. J Palliat Care 1991;7:6-9.

25. Fletcher CM (Chairman): Standardised questionnaire on respiratory symptoms: A statement prepared and approved by the MRC Committee on the Aetiology of Chronic Bronchitis (MRC breathlessness score). BMJ 1960;2:1665.

26. Carlucci A, Guerrieri A, Nava S: Palliative care in COPD patients: Is it only an end-of-life issue? Eur Respir Rev 2012;21:347-354.

27. Benzo R, Siemion W, Novotny P, et al.: Factors to inform clinicians about the end of life in severe chronic obstructive pulmonary disease. J Pain Symptom Manage 2013;46:491499.

28. Siouta N, Van Beek K, Preston N, et al.: Towards integration of palliative care in patients with chronic heart failure and chronic obstructive pulmonary disease: A systematic literature review of European guidelines and pathways. BMC Palliat Care 2016;15:18.

29. Vermylen JH, Szmuilowicz E, Kalhan R: Palliative care in COPD: An unmet area for quality improvement. Int J Chron Obstruct Pulmon Dis 2015;10:1543-1551.

30. Horton R, Rocker G, Dale A, et al.: Implementing a palliative care trial in advanced COPD: A feasibility assessment (the COPD IMPACT study). J Palliat Med 2013;16:67-73.

31. Burge PS, Calverley PM, Jones PW, et al.: Randomised, double blind, placebo controlled study of fluticasone propionate in patients with moderate to severe chronic obstructive pulmonary disease: The ISOLDE trial. BMJ 2000; 320:1297-1303.

32. Calverley PM, Anderson JA, Celli B, et al.: Salmeterol and fluticasone propionate and survival in chronic obstructive pulmonary disease. N Engl J Med 2007;356:775-789.

33. Tashkin DP, Celli B, Senn S, et al.: A 4-year trial of tiotropium in chronic obstructive pulmonary disease. N Engl J Med 2008;359:1543-1554.

34. Zielinski J, MacNee W, Wedzicha J, et al.: Causes of death in patients with COPD and chronic respiratory failure. Monaldi Arch Chest Dis 1997;52:43-47.

35. Garcia-Aymerich J, Barreiro E, Farrero E, et al.: Patients hospitalized for COPD have a high prevalence of modifiable risk factors for exacerbation (EFRAM study). Eur Respir J 2000;16:1037-1042.

36. Weingaertner V, Scheve C, Gerdes V, et al.: Breathlessness, functional status, distress, and palliative care needs over time in patients with advanced chronic obstructive pulmonary disease or lung cancer: A cohort study. J Pain Symptom Manage 2014;48:569-581. 
37. Blinderman CD, Homel P, Billings JA, et al.: Symptom distress and quality of life in patients with advanced chronic obstructive pulmonary disease. J Pain Symptom Manage 2009;38:115-123.

38. Ekstrom MP, Bornefalk-Hermansson A, Abernethy AP, Currow DC: Safety of benzodiazepines and opioids in very severe respiratory disease: National prospective study. BMJ 2014;348:g445.

39. Currow DC, McDonald C, Oaten S, et al.: Once-daily opioids for chronic dyspnea: A dose increment and pharmacovigilance study. J Pain Symptom Manage 2011;42: 388-399.

40. Poole PJ, Veale AG, Black PN: The effect of sustainedrelease morphine on breathlessness and quality of life in severe chronic obstructive pulmonary disease. Am J Respir Crit Care Med 1998;157(6 Pt 1):1877-1880.

41. Ecenarro PS, Iguiñiz MI, Tejada SP, et al.: Management of COPD in end-of-life care by Spanish pulmonologists. COPD 2018;15:171-176.

42. Duenk RG, Verhagen C, Dekhuijzen P, et al.: The view of pulmonologists on palliative care for patients with COPD: A survey study. Int J Chron Obstruct Pulmon Dis 2017;12: 299-311.

43. Miravitlles M, Soriano JB, García-Río F, et al.: Prevalence of COPD in Spain: Impact of undiagnosed COPD on quality of life and daily life activities. Thorax 2009;64: 863-868.

44. Boland J, Owen J, Ainscough R, Mahdi H: Developing a service for patients with very severe chronic obstructive pulmonary disease (COPD) within resources. BMJ Support Palliat Care 2014;4:196-201.

45. Cohen J, Beernaert K, Van den Block L, et al.: Differences in place of death between lung cancer and COPD patients: A 14-country study using death certificate data. NPJ Prim Care Respir Med 2017;27:14.

46. Royal College of Physicians, British Thoracic Society, British Lung Foundation: Report of the National COPD Audit 2008: Resources and Organisation of Care in Acute NHS Units Across the UK. London, United Kingdom: Royal College of Physicians, British Thoracic Society, British Lung Foundation, 2008.
47. Sinclair C, Auret KA, Evans SF, et al.: Advance care planning uptake among patients with severe long disease: a randomised patient preference trial of a nurse-led, facilitated advance care planning intervention. BMJ Open 2017; 7:e013415.

48. Corbelli J, Zhang D, Dionne-Odom JN, et al.: Association between palliative care and patient and caregiver outcomes: A systematic review and meta-analysis. JAMA 2016;316: 2104-2114.

49. Tavares N, Jarrett N, Hunt K, et al.: Palliative and end-oflife care conversations in COPD: A systematic literature review. ERJ Open Res 2017;3:00068-2016.

50. Mercadante S, Porzio G, Valle A, et al.: Palliative sedation in patients with advanced cancer followed at home: A systematic review. J Pain Symptom Manage 2011;41:754760 .

51. Nabal M, Palomar C, Juvero MT, et al.: Sedación paliativa: Situación actual y áreas de mejora. Rev Calid Asist 2014; 29:104-111.

52. Caraceni A, Zecca E, Martini C, et al.: Palliative sedation at the end of life at a tertiary cancer center. Support Care Cancer 2012;20:1299-1307.

53. Cohen J, Annemans L: Resource use and health care costs of COPD patients at the end of life: A systematic review. J Pain Symptom Manage 2016;52:588-599.

54. Bainbridge D, Seow H, Sussman J: Common components of efficacious in-home end-of-life care programs: A review of systematic reviews. J Am Geriatr Soc 2016;64:632-639.

55. Shepperd S, Gonçalves-Bradley DC, Straus SE, Wee B: Hospital at home: Home-based end-of-life care. Cochrane Database Syst Rev 2016;2:CD009231.

Address correspondence to:

Daniel Gainza-Miranda, MD

Equipo de soporte atención dani ciliaria área Norte de Madrid Avenida de España $\mathrm{ZO}$ San Sebastián de los Reyes 28701 Spain

E-mail:dgainza99@me.com 